\title{
Conhecimento dos acadêmicos de odontologia sobre traumatismos em dentes decíduos
}

\author{
Knowledge of dentistry's students on traumatisms \\ in deciduous teeth
}

\section{Gustavo Silva Peres ${ }^{1}$, Joana Vitória Batista Costa Melo², Éllen Maria Matos de Andrade², Daniela Nunes Nogueira ${ }^{4}$, Márcia Regina Soares Cruz ${ }^{5}$, Neusa Barros Dantas Neta ${ }^{6}$}

${ }^{1}$ Autor para correspondência. Faculdade Facid|Wyden. Teresina, Piauí, Brasil. ORCID: 0000-0002-1564-9502. gustavoceni2011@hotmail.com ${ }^{2}$ Faculdade Facid|Wyden. Teresina, Piauí, Brasil. ORCID: 0000-0001-9706-1798. joanavitoria_@hotmail.com 3Instituto Superior de Educação de Caxias. Caxias, Maranhão, Brasil. ORCID: 0000-0002-7676-6956. ellenmaria.odonto@gmail.com ${ }^{4}$ Faculdade Facid|Wyden. Teresina, Piauí, Brasil. ORCID: 0000-0003-0904-4694. daielanunes@hotmail.com ${ }^{5}$ Faculdade Facid |Wyden. Teresina, Piauí, Brasil. ORCID: 0000-0001-5633-0090. marciarscruz@hotmail.com ${ }^{6}$ Faculdade Facid|Wyden. Teresina, Piauí, Brasil. ORCID: 0000-0001-7127-1463. nbdn2@msn.com

RESUMO | INTRODUÇÃO: O trauma dentário é o conjunto de impactos que afeta os dentes e suas estruturas de suporte desde fratura em esmalte até a perda definitiva do elemento dentário. Entre os principais fatores etiológicos de traumatismo dentário na infância encontram-se as práticas desportivas, acidentes automobilísticos, atividades pertinentes à infância, agressões e fatores predisponentes individuais. O prognóstico das lesões traumáticas é influenciado pelo tipo e gravidade da lesão, intervalo de tempo entre o episódio do trauma e o tratamento inicial. OBJETIVO: Analisar o conhecimento dos acadêmicos de odontologia de uma instituição de ensino superior sobre trauma dentário em dentes decíduos. MATERIAIS E MÉTODOS: Trata-se de uma pesquisa transversal. Os dados foram coletados através da aplicação de um questionário, com perguntas sobre conhecimento em trauma dental e a conduta frente a situações de traumatismo dentário, realizado com alunos que cursaram a disciplina de Clínica Integrada Infantil I, II e III. Foi realizado análise descritiva dos dados e aplicado o Teste de Tendência Linear, considerando valor de $p<0,05$. RESULTADOS: Observou-se um baixo nível de conhecimento dos acadêmicos sobre o tema abordado, apenas $8 \%$ acertaram todas as questões, com a menor quantidade de acertos em casos de subluxação (37,3\% de acertos) e avulsão (41,2\% de acertos). Não houve associação do período com nível de conhecimento (valor de $p>0,05$ ). CONCLUSÃO: O conhecimento dos alunos sobre traumatismo dentário é baixo, principalmente sobre tratamento de casos de trauma como subluxação e avulsão.

PALAVRAS-CHAVE: Conhecimento. Traumatismo dentário. Odontopediatria.
ABSTRACT | INTRODUCTION: Dental trauma is the set of impacts that affect the teeth and their supporting structures from enamel fracture to the definitive loss of the dental element. Among the main etiological factors of childhood dental trauma are sports practices, car accidents, child-related activities, aggressions and individual predisposing factors. The prognosis of traumatic lesions is influenced by the type and severity of the injury, the time interval between the trauma episode and the initial treatment. OBJECTIVE: To analyze the knowledge of dentistry academics of a higher education institution on dental trauma in primary teeth. MATERIAL AND METHODS: This is a transversal research. The data were collected through the application of a questionnaire, with questions about knowledge in dental trauma and the conduct of dental trauma, carried out with students who attended the discipline of Integrated Children's Clinic I, II and III. A descriptive analysis of the data was performed and the Linear Trend Test was applied, considering $p$ value <0.05. RESULTS: A low level of academic knowledge about the subject was observed, only $8 \%$ answered all questions, with the least amount of correct answers in cases of subluxation (37.3\% of correct answers) and avulsion (41.2\% of correct answers). There was no association of the period with level of knowledge ( $p$ value> 0.05). CONCLUSION: The students' knowledge about dental trauma is low, mainly in the treatment of cases of trauma, such as subluxation and avulsion.

KEYWORDS: Knowledge. Tooth injuries. Pediatric dentistry. 


\section{Introdução}

As injúrias traumáticas apresentam alta prevalência dentre as lesões que atingem os tecidos bucais. É considerado traumatismo dentário toda lesão de natureza térmica, química ou física que afetam um dente. O trauma dentário interfere na qualidade de vida dos indivíduos afetados, seja por problemas de fala ou comprometimento da estética, fatores aos quais podem levar a problemas psicológicos e de convívio na sociedade'. Sabe-se que extensão e complexidade das lesões traumáticas dependem da natureza, da intensidade e características do dente envolvido².

As lesões traumáticas nos tecidos de sustentação estão envolvidas na maioria dos traumatismos que ocorre na dentição decídua, pois há alta prevalência de deslocamentos dentários em razão da maior resiliência do osso alveolar em crianças de baixa idade e da anatomia da raiz, que é menor e cuneiforme. Algumas dessas apresentam poucos sinais e sintomas, como a subluxação e luxação lateral. As luxações intrusiva, extrusiva e avulsão são consideradas lesões mais graves. As lesões das estruturas ósseas são caracterizadas pelo rompimento ou por fraturas das paredes alveolares, processo alveolar, fratura mandibular e fratura maxilar ${ }^{3}$.

O trauma dentário é responsável por cerca de $5 \%$ de todas as lesões que levam a tratamento ambulatorial ou hospitalar, sendo a região oral a sexta mais frequentemente afetada do corpo, especialmente na idade de 0 a 6 anos $^{4}$. Logo, por ser muito frequente os casos de traumatismo dentário de dentes decíduos, torna imprescindível que o cirurgião dentista esteja apto a atender estes casos, de modo a transmitir tranquilidade ao paciente e a seus familiares ${ }^{5}$.

Assim, é importante que os profissionais de Odontologia sejam avaliados quanto ao seu comportamento diante de traumas dentários, visando identificar as principais deficiências relacionadas com esta questão, para que seja possível o desenvolvimento de estratégias para melhorar o conhecimento dos profissionais perante esses casos $^{6}$.
Nesse contexto, o objetivo geral desse estudo foi identificar o conhecimento dos acadêmicos de Odontologia de uma instituição de ensino superior (IES) sobre trauma dentário em dentes decíduos. Além disso, objetivou-se verificar o conhecimento dos alunos sobre injúrias dentárias correlacionando com o tipo de trauma, caracterizar variáveis socioeconômicas e identificar o período do curso ao qual os alunos têm maior nível de conhecimento sobre o tema.

\section{Material e Método}

\section{Procedimentos Éticos}

O estudo foi desenvolvido de acordo com as exigências das Resoluções do Conselho Nacional de Saúde $n^{\circ} 466 / 2012$ e n510/2016, que tratam de pesquisas em seres humanos, mantendo em sigilo as informações que possam identificar os participantes da pesquisa.

A pesquisa foi submetida ao Comitê de Ética e Pesquisa (CEP) da FACID/Wyden, via Plataforma Brasil e obteve parecer favorável de №67049317.8.0000.5211. Os dados foram coletados após a aprovação pelo CEP. Os participantes foram devidamente esclarecidos da pesquisa e assinaram o Termo de Consentimento Livre e Esclarecido (TCLE), em duas vias.

Houve risco mínimo com o desenvolvimento da pesquisa, relacionado ao constrangimento dos participantes por não saberem responder as questões. Para minimizar esse risco houve aplicação do questionário de forma individual.

\section{Método da Pesquisa}

Trata-se de um estudo transversal, com uma abordagem quantitativa e descritivo quanto aos objetivos. 


\section{Cenário e Participantes do Estudo}

Teresina é a Capital do Piauí, possui 5 faculdades de Odontologia, com 1 faculdade pública federal e 4 universidades particulares. A pesquisa foi desenvolvida em uma instituição privada de ensino superior em Teresina-PI. A amostra foi do tipo censitária constituída por 102 alunos do $8^{\circ}, 9^{\circ}$ e $10^{\circ}$ período, que estavam cursando as disciplinas de Clínica Integrada Infantil I $(n=50)$, II $(n=42)$ ou III ( $n=41)$ (Tabela 1$)$.

\section{Coleta de Dados}

Os dados foram colhidos entre os meses de fevereiro e março de 2018, através da aplicação de questionário estruturado e padronizado, elaborado pelos pesquisadores em uma IES de Teresina, baseado no Guia The Management of Traumatic Dental Injuries: 3. Injuries in the Primary Dentition publicado pela International Association for Dental Traumatology (IADT) destinado aos participantes, com perguntas sobre conhecimento em trauma dental e a conduta frente a situações de trauma dentário ${ }^{7}$.

Os alunos foram abordados individualmente, não foi estabelecido tempo para responder o questionário.
Os questionários foram aplicados por uma única pessoa, na própria instituição de ensino dos estudantes.

\section{Organização e Análise dos Dados}

Os dados foram analisados usando Statistical Package for the Social Sciences(R)(SPSS(R), Chicago, IL, EUA.), Versão 20.0. Realizou-se análise descritiva dos dados, apresentando-os com frequências e porcentagens. Realizou-se o teste de Tendência Linear para associar variáveis dependentes com independentes. Considerou-se como significante valores de $p<0,05$.

\section{Resultados}

Este estudo foi realizado com 102 alunos, todos os convidados $(100,0 \%)$, aceitaram participar da pesquisa. A tabela 1 apresenta o perfil sociodemográfico dos participantes. De acordo com os resultados obtidos, 62,7 $\%$ dos acadêmicos que cursam os três últimos períodos do curso de Odontologia são mulheres, possuem renda familiar entre 3 a 4 salários mínimos (51\%), cursam o $8^{\circ}$ período (36,3\%) e têm idade de 22 anos (32,4\%), sendo a mínima de 19 anos e máxima de 38 anos.

Tabela 1. Descrição socioeconômica dos acadêmicos de Odontologia do oitavo ao décimo período (n=102)

\begin{tabular}{|c|c|c|c|}
\hline & & 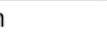 & $\%$ \\
\hline \multirow{2}{*}{ Sexo } & Masculino & 38 & 37,3 \\
\hline & Feminino & 64 & 62,7 \\
\hline \multirow{3}{*}{ Renda } & $1 \mathrm{~A} 2 \mathrm{SM}$ & 9 & 8,8 \\
\hline & $3 \mathrm{~A} 4 \mathrm{SM}$ & 52 & 51,0 \\
\hline & $\geq 5 \mathrm{SM}$ & 41 & 40,2 \\
\hline \multirow{3}{*}{ Período } & 8 & 37 & 36,3 \\
\hline & 9 & 35 & 34,3 \\
\hline & 10 & 30 & 29,4 \\
\hline \multirow{8}{*}{ Idade (anos) } & 19 & 1 & 1,0 \\
\hline & 20 & 9 & 8,8 \\
\hline & 21 & 22 & 21,6 \\
\hline & 22 & 33 & 32,4 \\
\hline & 23 & 15 & 14,7 \\
\hline & 24 & 12 & 11,8 \\
\hline & $\geq 25$ anos & 10 & 9,8 \\
\hline & Total & 102 & 100,0 \\
\hline
\end{tabular}


O gráfico 1 apresenta o nível de conhecimento sobre trauma dentário de dentes decíduos obtidos pelos acadêmicos que já cursaram ou cursam a disciplina de clínica integrada infantil. Conforme os dados obtidos apenas 8,8\% dos estudantes possuem conhecimento pleno sobre traumas dentários em dentes decíduos e 2,9\% dos estudantes acertaram apenas 1 pergunta sobre o tema.

Gráfico 1. Percentagem de estudantes que responderam corretamente as questões sobre os traumas dentários em dentes decíduos

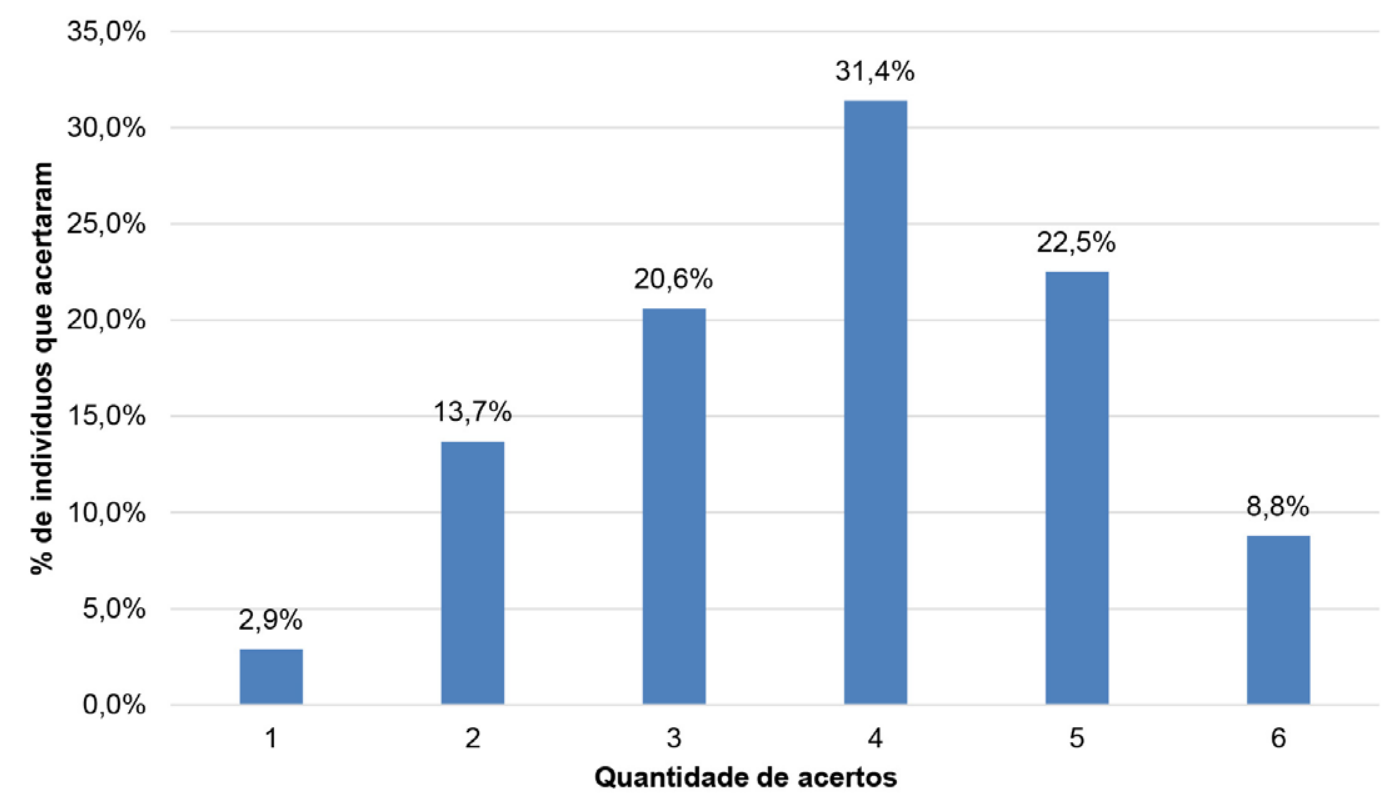

O nível de acertos dos estudantes levando em consideração o assunto abordado em cada questão foram dispostos no Gráfico 2. Dentre os assuntos abordados, a subluxação e avulsão tiveram menor frequência de conhecimento de $37,3 \%$ e $41,2 \%$, respectivamente.

Gráfico 2. Porcentagem de estudantes de odontologia que acertaram as respostas sobre os traumas dentários em dentes decíduos distribuídos por questão

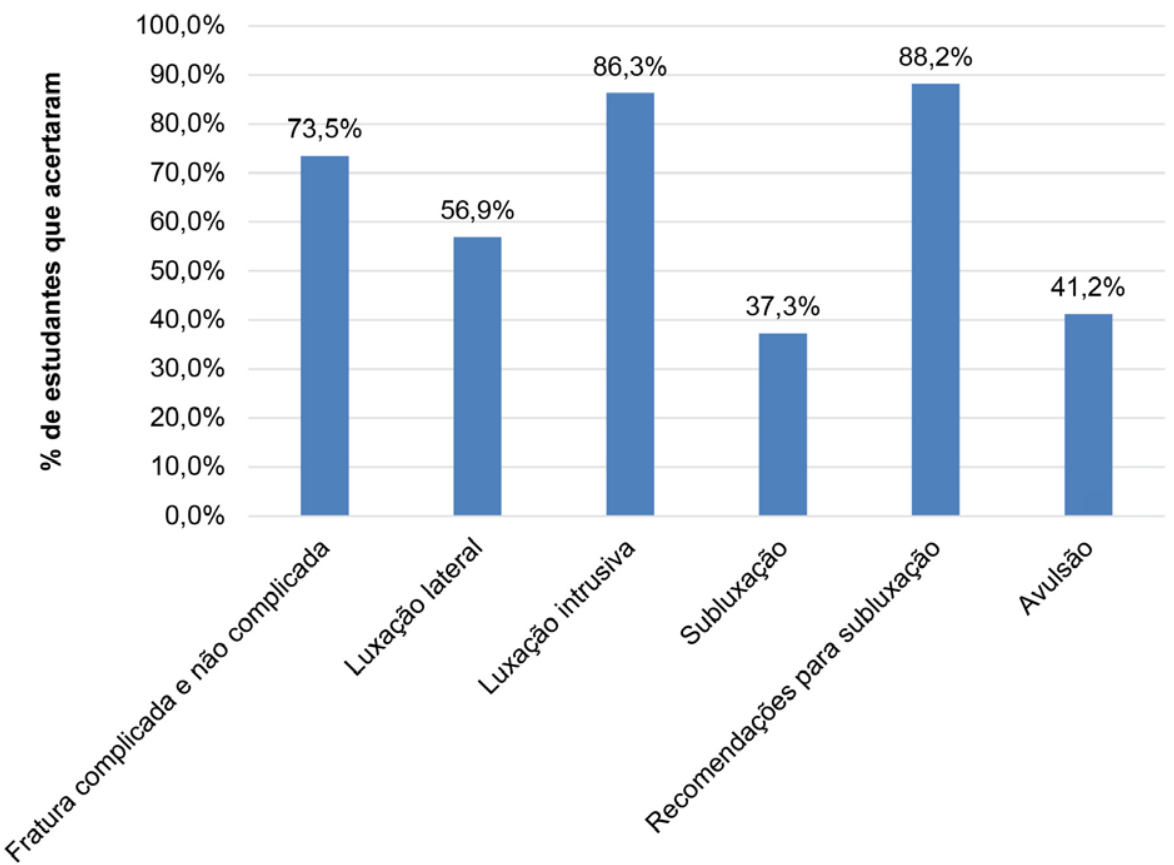

Questões sobre trauma 
$\mathrm{Na}$ tabela 2 analisou a presença de associação entre questões respondidas corretamente com as variáveis sexo, renda e período dos alunos participantes da pesquisa. Conforme os resultados identificados no estudo dos alunos que acertaram todas as perguntas sobre trauma dentário em dentes decíduos, $66,7 \%$ eram do $8^{\circ}$ período, 55,6\% possuem renda familiar maior ou igual a 5 salários mínimos e $66,7 \%$ dos estudantes eram do sexo feminino. Entretanto, não houve associação entre quantidade de questões certas sobre trauma dentário de dentes decíduos com sexo, renda e período (valor de $p>0,05$ ).

Tabela 2. Associação entre quantidade de questões certas sobre traumas dentários de dentes decíduos com sexo, renda e período (n=102)

\begin{tabular}{|c|c|c|c|c|c|c|c|}
\hline & \multicolumn{6}{|c|}{ Total de questões certas } & \multirow{2}{*}{$\begin{array}{l}\text { Valor } \\
\text { de } p^{*}\end{array}$} \\
\hline & 1 & 2 & 3 & 4 & 5 & 6 & \\
\hline \multicolumn{8}{|l|}{ Sexo } \\
\hline \multirow{2}{*}{ Masculino } & 2 & 5 & 7 & 13 & 8 & 3 & 0,686 \\
\hline & $66,7 \%$ & $35,7 \%$ & $33,3 \%$ & $40,6 \%$ & $34,8 \%$ & $33,3 \%$ & \\
\hline \multirow{2}{*}{ Feminino } & 1 & 9 & 14 & 19 & 15 & 6 & \\
\hline & $33,3 \%$ & $64,3 \%$ & $66,7 \%$ & $59,4 \%$ & $65,2 \%$ & $66,7 \%$ & \\
\hline \multicolumn{8}{|l|}{ Renda } \\
\hline \multirow{2}{*}{$1 \mathrm{~A} 2 \mathrm{SM}$} & 0 & 1 & 3 & 4 & 0 & 1 & 0,532 \\
\hline & $0,0 \%$ & $7,1 \%$ & $14,3 \%$ & $12,5 \%$ & $0,0 \%$ & $11,1 \%$ & \\
\hline \multirow{2}{*}{$3 \mathrm{~A} 4 \mathrm{SM}$} & 1 & 10 & 8 & 16 & 14 & 3 & \\
\hline & $33,3 \%$ & $71,4 \%$ & $38,1 \%$ & $50,0 \%$ & $60,9 \%$ & $33,3 \%$ & \\
\hline \multirow{2}{*}{$>=5 \mathrm{SM}$} & 2 & 3 & 10 & 12 & 9 & 5 & \\
\hline & $66,7 \%$ & $21,4 \%$ & $47,6 \%$ & $37,5 \%$ & $39,1 \%$ & $55,6 \%$ & \\
\hline \multicolumn{8}{|l|}{ Período } \\
\hline \multirow{2}{*}{8} & 1 & 4 & 7 & 10 & 9 & 6 & 0,244 \\
\hline & $33,3 \%$ & $28,6 \%$ & $33,3 \%$ & $31,2 \%$ & $39,1 \%$ & $66,7 \%$ & \\
\hline \multirow{2}{*}{9} & 1 & 6 & 7 & 13 & 6 & 2 & \\
\hline & $33,3 \%$ & $42,9 \%$ & $33,3 \%$ & $40,6 \%$ & $26,1 \%$ & $22,2 \%$ & \\
\hline \multirow{2}{*}{10} & 1 & 4 & 7 & 9 & 8 & 1 & \\
\hline & $33,3 \%$ & $28,6 \%$ & $33,3 \%$ & $28,1 \%$ & $34,8 \%$ & $11,1 \%$ & \\
\hline \multirow{2}{*}{ Total } & 3 & 14 & 21 & 32 & 23 & 9 & \\
\hline & $100,0 \%$ & $100,0 \%$ & $100,0 \%$ & $100,0 \%$ & $100,0 \%$ & $100,0 \%$ & \\
\hline
\end{tabular}

*Teste de Tendência Linear

\section{Discussão}

O estudo foi desenvolvido em IES com alunos dos últimos períodos do curso de Odontologia. De acordo com os resultados obtidos no estudo, houve uma maior frequência de participantes do sexo feminino, com renda familiar entre 3 a 4 salários, com idade de 22 anos.

Corroborando com os resultados, em um estudo realizado por Ferraz et al. (2018), que trata sobre o perfil dos estudantes do curso de Odontologia, a maioria é do gênero feminino (54\%) e a faixa etária predominante foi entre 20 e 30 anos (60\%)8. Em relação à condição socioeconômica das famílias dos estudantes do curso, resultados semelhantes foram encontrados por Querino e colaboradores (2018), em que a maioria das famílias analisadas ganham entre 3 e 5 salários mínimos $(41,6 \%)^{9}$. 
A Odontologia, historicamente, foi caracterizada como uma profissão tipicamente masculina. Entretanto, tem-se discutido que ela passa por um processo crescente de feminização, que se refere, normalmente, a um franco crescimento da população feminina em algumas profissões que historicamente eram desempenhadas pelos homens, como exemplo Medicina e Odontologia ${ }^{10}$. Conhecer o perfil do profissional norteia mudanças na profissão, novas tendências e necessidades do mercado de trabalho, além de orientar futuras decisões dos gestores do curso ${ }^{8}$.

Ao se avaliar o conhecimento sobre traumatismo dentário em dentes decíduos, nesse estudo verificou-se que ele foi baixo, pois poucos alunos acertaram todos as questões. Um estudo semelhante a esse, mas realizado com dentistas mostrou um baixo nível de conhecimento quanto ao tratamento de urgência em lesões dentárias por trauma. De acordo com o autor isso pode ser explicado porque o currículo odontológico tradicional apresenta oportunidades limitadas para os alunos avaliarem e tratarem pacientes com traumatismo dentário. Como resultado dessa falta de exposição, a maioria dos dentistas é pouco preparado para gerenciar trauma dentário ${ }^{6}$.

O tratamento da lesão dentária traumática pode ser relativamente complexo e, muitas vezes, requer diferentes especialidades odontológicas. Tais lesões, são consideradas como um sério problema de saúde pública, devido à alta taxa de prevalência entre crianças, 32,9\% e adolescentes $28,2 \%$, e seu impacto na qualidade de vida relacionada à saúde bucal em termos de bem-estar social e emocional, 35,5\% e 34,9\% respectivamente ${ }^{11}$.

Os dentes decíduos desempenham um papel importante no alinhamento adequado, espaçamento e oclusão dos dentes permanentes ${ }^{12}$. O prognóstico das lesões traumáticas é influenciado pelo tipo e gravidade da lesão, intervalo de tempo entre o episódio do trauma e o tratamento inicial. As lesões devem ser tratadas imediatamente, para evitar dor e complicações futuras. O cirurgião-dentista e os pais devem ser conscientizados da importância do acompanhamento clínico e radiográfico dos traumatismos dentários ${ }^{13,14}$. Desta forma é um desafio para o profissional que necessita ter um adequado manejo do paciente, conhecimento técnico-científico para realizar um diagnóstico correto, a fim de, planejar um correto e eficaz tratamento ${ }^{13}$.
Portanto, a deficiência de conhecimento observada nos alunos que participaram do estudo, pode ser considerada um problema, uma vez que o traumatismo dentário é uma situação de urgência odontológica e requer conhecimento por parte do profissional, sendo fundamental a implementação de medidas que melhore a formação dos acadêmicos sobre trauma dentário.

O nível de acertos dos estudantes levando em consideração o assunto abordado foi considerado baixo para os temas subluxação e avulsão e alto para luxação intrusiva. Uma possível explicação para esse resultado seria o confundimento dos alunos entre os protocolos de tratamento de avulsão e subluxação de dentes decíduos pelo protocolo de dentes permanentes. $O$ tratamento de avulsão consiste em não reimplantar o dente decíduo pela possibilidade de lesão do dente permanente e realização de reabilitação protética. Já para a subluxação deve ser realizada a orientação dietética, restrição do uso de chupetas e mamadeiras e em caso de mobilidade acentuada uso de contenção flexível ou semi-rígida por 10 a 14 dias? .

Neste estudo não houve associação entre quantidade de questões certas sobre trauma dentário de dentes decíduos com sexo, renda e período (valor de $p>0,05)$. Entretanto, dentre os alunos que acertaram todas as perguntas sobre trauma dentário em dentes decíduos, a maior frequência encontrada foi no $8^{\circ}$ período, entre os que possuíam renda familiar maior ou igual a 5 salários mínimos e eram do sexo feminino.

O assunto trauma em dentes decíduos é visto como último conteúdo no $7^{\circ}$ período. O conteúdo trauma em dentes decíduos nos outros períodos é visto como forma de seminário, também no final do semestre. A aplicação do questionário foi realizada pouco tempo depois dos alunos do $8^{\circ}$ período terem visto o conteúdo no $7^{\circ}$ período. Assim, esse pode ter sido um motivo para maior frequência de alunos acertando todas as questões no $8^{\circ}$ período.

Segundo Buldur e Kapdan (2018), o nível de conhecimento dos dentistas varia de acordo com o tipo de lesão dentária traumática. O baixo nível de conhecimento dos dentistas sobre trauma dentário indicam a necessidade de programas de educação continuada para os profissionais nesse assunto ${ }^{15}$. Como a gestão de lesões traumáticas é importante 
e o conhecimento sobre este assunto está em constante mudança, é necessário um planejamento adequado para atualizar o conhecimento dos dentistas gerais sobre o tema ${ }^{3}$.

A presença de profissionais treinados em lesões traumáticas dentária em instituições e policlínicas que prestam serviços de tratamento de emergência podem melhorar o prognóstico dos tratamentos, proporcionando uma intervenção imediata e correta aos dentes traumatizados ${ }^{15}$.

A taxa de falha no tratamento de fraturas simples da coroa é motivo de preocupação porque estas são geralmente consideradas pequenas lesões dentárias. É importante que todos os tecidos adjacentes ao trauma sejam examinados (não apenas a fratura dentária), para que não ocorram complicações futuras ${ }^{16}$. As decisões sobre o tratamento ideal para cada criança requerem conhecimento sobre chance de cura e o risco de complicações subsequentes ${ }^{17}$.

O traumatismo alvéolo dentário na dentição decídua poderá acarretar problemas na dentição sucessora permanente em desenvolvimento,além de trazer sérias consequências, pois há uma relação anatômica íntima entre os ápices dos dentes decíduos e os germes dos permanentes ${ }^{18}$.

A partir dos dados encontrados no estudo, pode-se sugerir que sejam implementadas medidas para meIhorar a habilitação dos alunos, como por exemplo a implementação de mais aulas, uso de metodologias ativas nas aulas, práticas mais amplas que inspirem o aluno sobre a importância de seu conhecimento, no tocante do manejo adequado do trauma dentário em infanto-juvenis, já que o assunto é abordado em forma de seminários e leitura de artigos científicos.

\section{Conclusão}

O conhecimento geral sobre traumatismo em dente decíduo obteve baixa prevalência de acerto. $\mathrm{O}$ assunto avulsão e subluxação foram os de menor conhecimento. Não houve diferença no nível de conhecimento entre o $8^{\circ}, 9^{\circ}$ e $10^{\circ}$ períodos.

\section{Contribuições dos autores}

Peres GS, Melo JVBC realizou a revisão de literatura e análise de dados e escreveu o artigo. Andrade EMM realizou a análise e discussão dos resultados. Nogueira DN e Cruz MRS criou o desenho do estudo, realizou a análise dos resultados e a avaliação crítica. Dantas Neta NB criou o desenho do estudo e realizou a análise e discussão dos resultados, avaliação crítica e orientação para o trabalho.

\section{Conflitos de interesses}

Nenhum conflito financeiro, legal ou político envolvendo terceiros (governo, empresas e fundações privadas, etc.) foi declarado para nenhum aspecto do trabalho submetido (incluindo mas não limitando-se a subvenções e financiamentos, participação em conselho consultivo, desenho de estudo, preparação de manuscrito, análise estatística, etc.).

\section{Referências}

1. Silva $A B$, Ferreira MC. Traumas dentários em escolares de 8 a 14 anos na Escola Monte Castelo / Passo Fundo-RS. J Oral Invest. 2014;3(2):13-8. doi: 10.18256/2238-510X/j.oralinvestigations. v3n2p13-18

2. Vieira EM, Cangussu MCT, Vianna MIP, Cabral MBB, Roque RN, Anjos ES. Prevalência, gravidade e fatores associados ao traumatismo dentário em escolares de 12 e 15-19 anos de idade em Salvador, Bahia. Rev Saúde Col UEFS. 2017;7(1):51-7. doi: 10.13102/rscdauefs.v7i1.1218

3. Losso EM, Tavares MCR, Bertoli FMP, Baratto Filho F. Traumatismo dentoalveolar na dentição decidua. Rev Sul-Bras Odontol. 2011;8(1):1-20.

4. Nakajima T, Iwase Y, Saitoh I, Nogami Y, Matsueda K, Hayasaki $\mathrm{H}$. Reconsidering the treatment plan for traumatized teeth - $\mathrm{A}$ case of lateral luxation with severe displacement. Ped Dent J. 2017;27(2):109-13. doi: 10.1016/j.pdj.2016.11.002

5. Fracasso MLC, Santin GC, Terra GMO, Martioli G, Provenzano MGA, Camilo A et al. Injúrias dentárias em dentes decíduos: estudo longitudinal. Rev Saúde Pesq. 2016;9(3):461-71. doi: 10.177651/1983-1870.2016v9n3p461-471

6. Azevedo RA, Corrêa MB, Torriani MA, Demarco FF, Lund RG. Treatment of dento-alveolar trauma: knowledge evaluation from southern Brazilian dentists. Braz J Oral Sci. 2016;15(4). doi: 10.20396/bjos.v15i4.8650044 
7. Malmgren B, Andreasen JO, Flores MT, Robertson A, DiAngelis AJ, Andersson L et al. Guidelines for the Management of Traumatic Dental Injuries: 3. Injuries in the Primary Dentition. Pediatr Dent. 2016 Oct;39(6):420-428. doi: 10.1111/j.16009657.2012.01146.x

8. Ferraz MAAL, Nolêto MSC, Martins LLN, Bandeira SRL, Portela SGC, Pinto PHV et al. Perfil dos egressos do curso de Odontologia da Universidade Estadual do Piauí. Rev ABENO. 2018;18(1):56-62. doi: $10.30979 /$ rev.abeno.v18i1.392

9. Querino JPFO, Peixoto LR, Sampaio GAM. Perfil dos concluintes de Odontologia da Universidade Estadual da Paraíba. Rev ABENO. 2018;18(1):170-81. doi: 10.30979/rev.abeno.v18i1.416

10. Costa SM, Durões SJA, Abreu MHNG. Feminização do curso de Odontologia da Universidade Federal de Montes Claros. Rev Ciênc Saúde Col. 2010;15(1):1865-73. doi: 10.1590/S1413$\underline{81232010000700100}$

11. Oliveira FS, Goursand D, Ferreira RC, Paiva PCP, Paiva HN, Ferreira EF et al. Traumatic dental injuries in Brazilian children and oral health related quality of life. Dent Traumatol. 2018;34(1):2835. doi: $10.1111 /$ edt.12358

12. Sajjadian N, Shajari H, Jahadi R, Barakat MG, Sajjadian A. Relationship between birth weight and time of first deciduous tooth eruption in 143 consecutively born infants. Pediatr Neonatol. 2010;51(4):235-7. doi: 10.1016/S1875-9572(10)60044-7

13. Francisco SS, Amaral RC, Vieira LMM, Braga CKP, Murrer RD. Conhecimento de estudantes de Educação Física de Juazeiro do Norte-CE sobre o atendimento emergencial ao trauma dental, avulsão e reimplante dental. J Health Sci Inst. 2016;34(2):75-81.

14. Rodrigues I, Silva BS, Ceron LC, Lopes TS, Venante HS, Santin GC et al. Tratamento da luxação lateral em dentes decíduos importância da proservação. Braz J Surg Clin Res. 2017;19(1):90-5.

15. Buldur B, Kapdan A. Factors associated with knowledge and attitude of management of traumatic dental injuries: a cross-sectional study among Turkish Dentists. Rev Pesq Bras Odontoped Clín Int. 2018;18(1):39-48. doi: 10.4034/ PBOCI.2018.181.30

16. Ritwik P, Massey C, Hagan J. Epidemiology and outcomes of dental trauma cases from an urban pediatric emergency department. Dent Traumatol. 2015;31(2):97-102. doi: 10.1111/ edt. 12148

17. Lauridsen $E$, Blanche $P$, Yousaf N, Andreasen JO. The risk of healing complications in primary teeth with extrusive or lateral luxation - A retrospective cohort study. Dent Traumatol. 2017;33(4):307-16. doi: 10.1111/edt.12340

18. Souza JGM, Lazzarin HC, Zeni BC, Filipin CL, Berft CL, Zilio TH. Conhecimento dos responsáveis das crianças atendidas na clínica odontológica da Unipar campus Cascavel - PR sobre traumatismo alvéolo dentário. Rev Odontol Clín Cient. 2018;17(1):39-44. 